\title{
Favorable response to combined androgen blockade for metastatic cutaneous apocrine carcinoma: a case report
}


Hiroshi Ariyama, MD, PhD ${ }^{a}$, Hitoshi Kusaba, MD, PhDa , Hidetaka Yamamoto, MD, PhD ${ }^{b}$, Yoshinao Oda, MD, PhD Koichi Akashi, MD, PhDª, Eishi Baba, MD, PhD ${ }^{c, t}$

\begin{abstract}
Introduction: Most cases of cutaneous apocrine carcinoma (CAC) express androgen receptor. Androgen signal is thought to have a relationship on the survival and cell growth of the tumor.

Presentation of Case: A 53-year-old man with a red superficial nodule on the left axilla and a back pain was diagnosed with CAC in the left axilla with multiple bone and bone marrow metastases. After cytotoxic chemotherapy for around 4 years, severe anemia, thrombocytopenia, and disseminated intravascular coagulation progressed. We started combined androgen blockade by bicaltamide and degarelix, and the therapy had successfully maintained stable disease for more than 6 months.

Discussion: Most cases of CAC express androgen receptor. Androgen signal could have a relationship on the survival and cell growth of the tumor.
\end{abstract}

Conclusion: Antiandrogen therapy for androgen-receptor positive CAC is a promising therapeutic option.

Keywords: Apocrine carcinoma, Androgen receptor, Antiandrogen therapy, Combination androgen blockade, Case report

Cutaneous apocrine carcinoma $(\mathrm{CAC})$ is a rare cutaneous adnexal cancer, occurring in 1 per 10 million people, according to the National Cancer Institute Surveillance, Epidemiology, and End Results (SEER) registry from 1973 to $2006^{[1]}$. CAC occurs equally in both sexes (males $52 \%$ and females $48 \%$ ), and the median age of onset is 67 years ${ }^{[1]}$. CAC often arises in the axilla and anogenital region, which are rich in apocrine glands. The causes and risk factors for CAC have not been clarified. The survival of patients with local/regional disease and metastatic disease has been reported to be 55.0 and 33.0 months, respectively ${ }^{[1]}$.

Most cases are diagnosed as local/regional disease, and they undergo surgical treatment. On the other hand, metastatic CAC is

\footnotetext{
${ }^{a}$ Department of Hematology, Oncology and Cardiovascular Disease, Kyushu University Hospital, Departments of ${ }^{b}$ Anatomic Pathology and ${ }^{C}$ Oncology and Social Medicine, Graduate School of Medical Sciences, Kyushu University, Fukuoka, Japan

This manuscript has been peer reviewed.
}

Sponsorships or competing interests that may be relevant to content are disclosed at the end of this article.

${ }^{*}$ Corresponding author. Address: Department of Oncology and Social Medicine, Graduate School of Medical Sciences, Kyushu University, 3-1-1 Maidashi, Higashi-ku, Fukuoka 812-8582, Japan. Tel: + 81-92-642 6921; fax: + 81-92-642-6922. E-mail address: e-baba@intmed1.med.kyushu-u.ac.jp (E. Baba).

Copyright @ 2020 The Authors. Published by Wolters Kluwer Health, Inc. on behalf of IJS Publishing Group Ltd. This is an open access article distributed under the Creative Commons Attribution-NoDerivatives License 4.0, which allows for redistribution, commercial and non-commercial, as long as it is passed along unchanged and in whole, with credit to the author.

International Journal of Surgery Oncology (2020) 5:e105

Received 5 August 2020; Accepted 10 October 2020

Published online 6 January 2021

http://dx.doi.org/10.1097//J9.0000000000000105 quite rare, and no standard treatment has been established. There are only a few reports of treatment for metastatic CAC. Several case studies described the use of cytotoxic agents including adriamycin, cyclophosphamide, vinblastine, bleomycin, 5-fluorouracil, and methotrexate ${ }^{[2-4]}$, and exploratory treatments such as antiHER2 antibody ${ }^{[5]}$ and PD-1 blockade were also reported ${ }^{[6]}$.

Increased expression of androgen receptor (AR) has been shown in $\mathrm{CAC}^{[7]}$, and androgen signaling is thought to be associated with tumor growth ${ }^{[8]}$. Although the potential benefit of antiandrogen therapy for CAC has been suggested, there have been no reports of antiandrogen therapy for CAC so far. In addition, while previous reports showed CAC with distant metastasis of lung ${ }^{[2]}$, brain ${ }^{[9]}$ and bone ${ }^{[10]}$, this is the first report of CAC with bone marrow metastasis. This is the first report which showed the efficacy of antiandrogen therapy for CAC.

\section{Case presentation}

A 53-year-old Asian man, who had no notable past medical or surgical history, had a 4-year history of a severe back pain. He did not take any medication. He had no notable family history of malignant tumor. In 2015, computed tomography (CT) showed osteoplastic changes of the vertebrae, and fluorodeoxyglucosepositron emission tomography/computed tomography (FDG-PET/CT) showed high FDG uptake by all vertebrae in the previous hospital (Fig. 1). He was referred to our hospital for further examination and treatment in 2015. A reddish, flat, and stiff nodule, with a diameter of $3 \mathrm{~cm}$ (Fig. 2), was observed in his left axilla. No obvious lesions were found in the mammary glands or salivary glands. Peripheral blood examination showed immature white and red blood cells, which suggested bone marrow involvement of the tumor. He had no notable past history and family history. Pathologic examination of a biopsy 

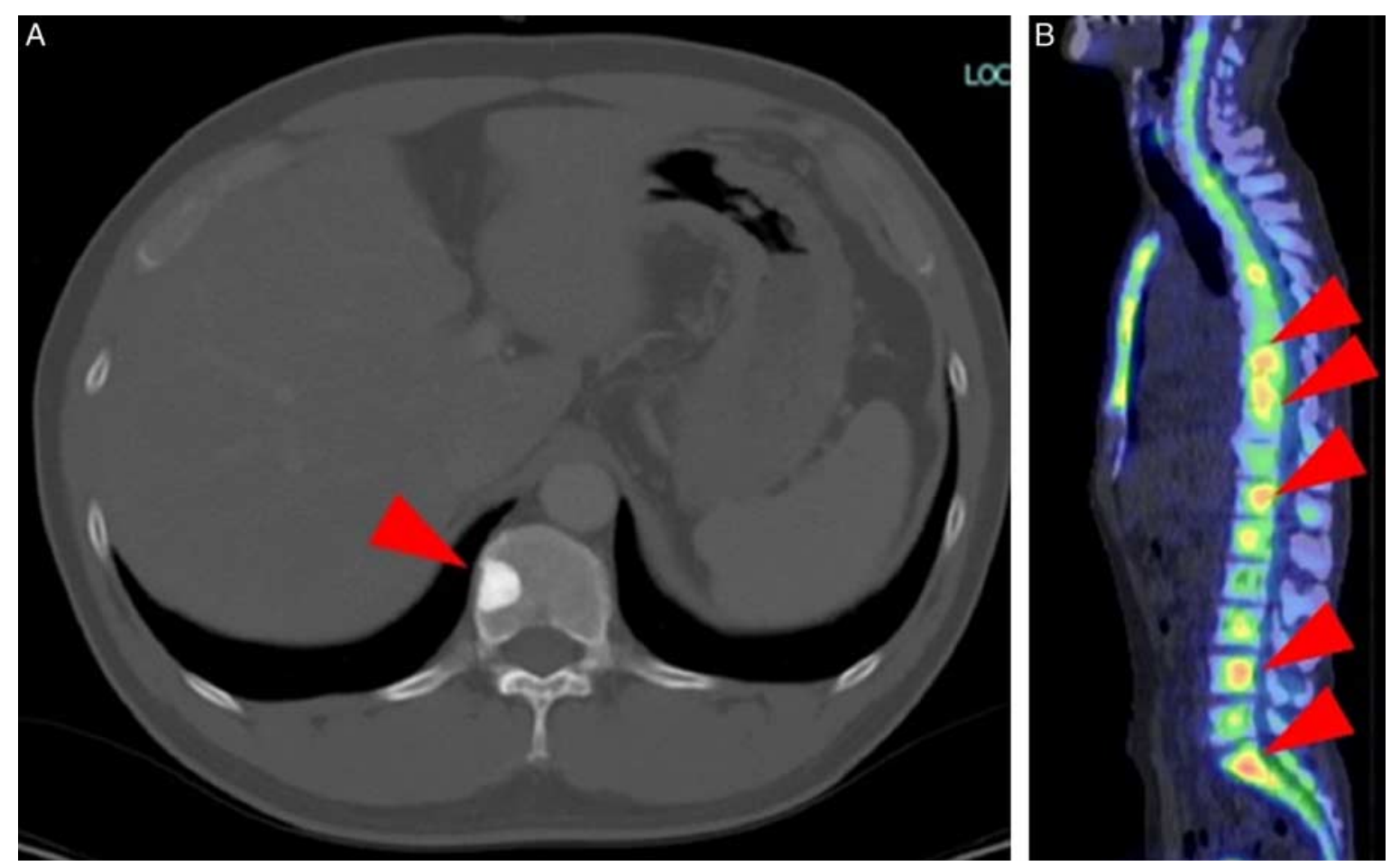

Figure 1. Computed tomography and fluorodeoxyglucose-positron emission tomography (FDG-PET) at the initial diagnosis in 2015. Arrow heads indicate metastatic sites of the spine (A) and high FDG uptake in the spine (B).

specimen of the nodule in his left axilla showed atypical cells having hyperchromatic nuclei and intracytoplasmic mucin in the dermis (Fig. 3A). Immunohistochemically, the cells were positive for cytokeratin-7 (CK7), gross cystic disease fluid protein 15 (GCDFP15), and AR (Fig. 3B), but negative for CK20, caudal type homeobox 2 (CDX2), thyroid transcription factor 1 (TTF-1), napsin A, estrogen receptor, and progesterone receptor. Human epidermal growth factor receptor 2 (HER2) was score $2+$, equivocal, according to the guideline of breast cancer in $2013^{[11]}$. These features suggested carcinoma with apocrine differentiation. Bone marrow biopsy showed carcinoma cells having eosinophilic cytoplasm and intracytoplasmic mucin with a glandular cavity structure, which suggested bone marrow metastasis of apocrine carcinoma (Figs. 3C, D). Because of those pathologic features and the finding that no lesion was found in other regions such as the mammary



Figure 2. The superficial nodule on the left axilla. glands or salivary glands, he was finally diagnosed with CAC with bone and bone marrow metastases.

The patient received tegafur/gimeracil/oteracil (S-1) treatment as a first-line therapy, and the disease was well controlled for 3 years. However, the treatment was changed to paclitaxel because of worsening anemia and thrombocytopenia. No significant change of the primary left axillary nodule was observed. After 7 months, anemia and thrombocytopenia caused by bone marrow metastases gradually progressed, and he needed transfusion of red blood cell and platelet components due to severe anemia (hemoglobin $4.7 \mathrm{~g} / \mathrm{dL}$ ) and thrombocytopenia (platelets $1.3 \times 10^{4} / \mu \mathrm{L}$ ). The blood examination also showed evidence of disseminated intravascular coagulation (DIC) (fibrinogen 205 $\mathrm{mg} / \mathrm{dL}$, prothrombin time-international normalization ratio (PT-INR) 1.24, fibrin/fibrinogen degradation products (FDP) 41.9 $\mu \mathrm{g} / \mathrm{mL}$, D-dimer $11.4 \mu \mathrm{g} / \mathrm{mL}$ ), suggesting progression of bone marrow metastases. He was admitted to our department for third-line treatment in April 2019. His Eastern Cooperative Oncology Group performance status was 2 . It was thought that he would not be able to tolerate cytotoxic chemotherapeutic agents. The genomic profiling examination (FoundationOne CDx assay; Foundation Medicine, Inc. Massachusetts) showed the activating mutation of PIK3CA gene, but no therapeutic options, including a clinical trial targeting PIK3CA mutation, were available.

Because the tumor was positive for $A R$, and there were some previous reports of the efficacy of antiandrogen therapy for ARpositive tumors, antiandrogen therapy was started. With the patient's written, informed consent, antiandrogen therapy using bicaltamide (80 mg per day, orally, continuous administration) and degarelix (240 mg every 4 weeks, subcutaneously) was initiated. Fourteen days after the initiation of the therapy, the anemia and thrombocytopenia 

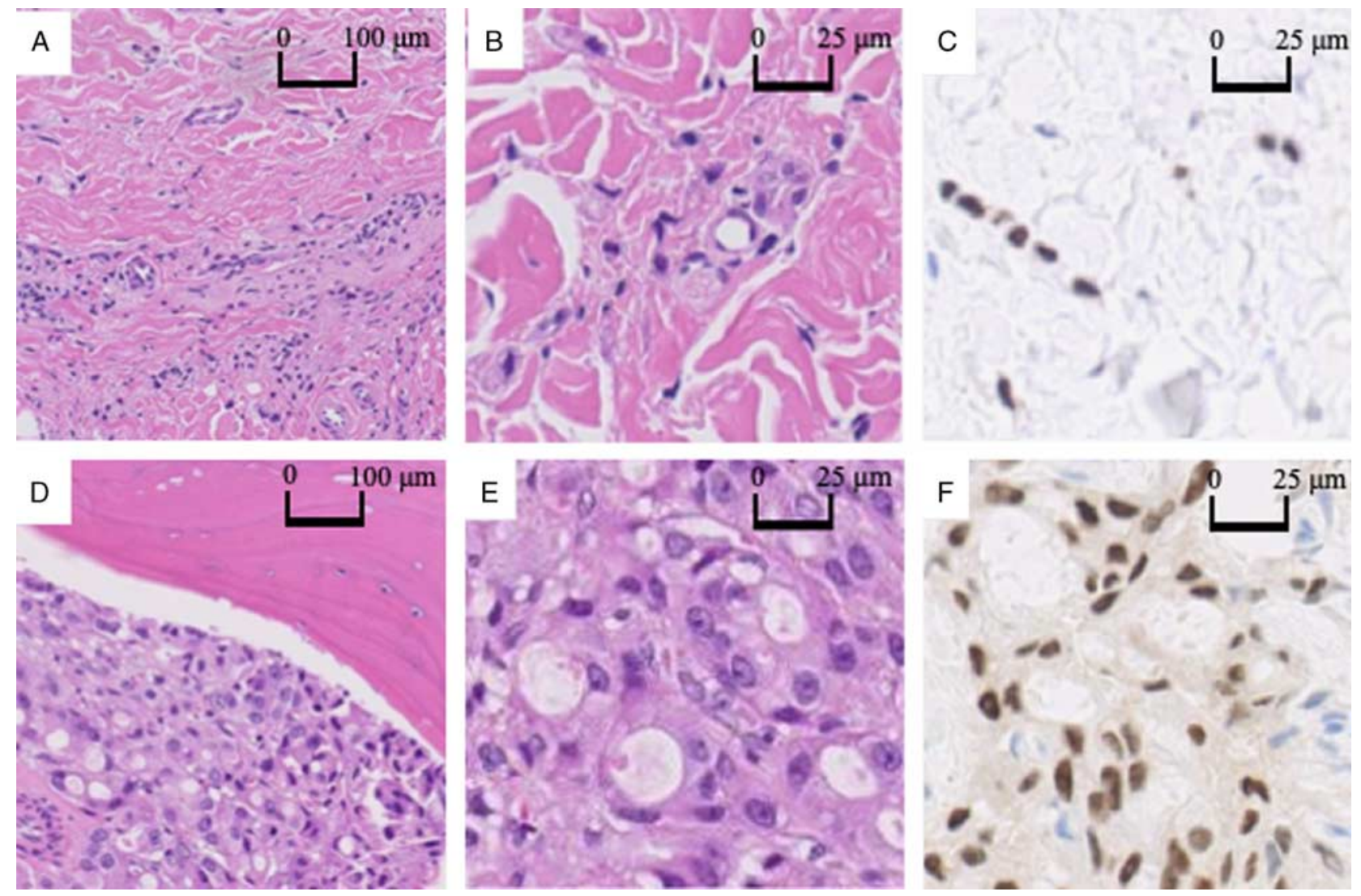

Figure 3. Histologic examination of the primary and metastatic site of the tumors. A, Hematoxylin and eosin (HE) staining of the primary tumor of low-magnification power $(\times 10)$. B, The same micrograph as (A) of high-magnification power $(\times 40)$. C, Immunostaining with anti-androgen receptor of the primary tumor. D, HE staining of the bone marrow metastasis of low-magnification power $(\times 10)$. E, The same micrograph as (D) of high-magnification power $(\times 40)$. F, Immunostaining with antiandrogen receptor of the bone marrow metastasis.

improved, to the extent that he did not need transfusion. His DIC status also improved (fibrinogen $253 \mathrm{mg} / \mathrm{dL}$, PT-INR 1.06, FDP $19.6 \mu \mathrm{g} / \mathrm{dL}$, D-dimer $4.0 \mu \mathrm{g} / \mathrm{dL}$ ), which strongly indicated a therapeutic response of the bone marrow metastases. He had an adverse event, an injection site reaction of grade 1 in the Common Terminology Criteria for Adverse Events (CTCAE). He has now been treated with the antiandrogen therapy for more than 6 months, without worsening of the anemia, thrombocytopenia, and DIC status. This case report is reported in line with the SCARE 2018 ${ }^{[12]}$.

\section{Discussion}

$\mathrm{CAC}$ is one of the adenocarcinomas originating from apocrine glands that mainly arises in the axilla and anogenital region, which are rich in apocrine glands. Although apocrine gland adenocarcinoma is reported to have a relatively nonaggressive nature, lymph node metastasis is found in $40 \%-50 \%$ of the patients at initial diagnosis ${ }^{[13,14]}$. Some reports showed that extranodal metastasis is rarely observed ${ }^{[14,15]}$, but liver, lung, and bone marrow metastases are known to occur ${ }^{[13,14]}$. Because metastatic CAC is rare, no standard treatment has been established. Several case reports and case series have described treatment for metastatic CAC using cytotoxic drugs ${ }^{[2-4]}$, anti-HER2 antibody ${ }^{[5]}$, and PD-1 inhibitor ${ }^{[6]}$.

The present case had CAC with bone and bone marrow metastases that caused severe anemia, thrombocytopenia, and
DIC. Cytotoxic chemotherapy agents, S-1 and paclitaxel, had been administered for $\sim 4$ years and provided disease control for a certain period. However, the patient developed drug resistance. Continuation of cytotoxic drugs was not suitable because of his life-threatening anemia and thrombocytopenia. Since antiandrogen therapy has less risk of myelosuppression, it was thought that the patient was more likely to tolerate it than cytotoxic drugs. The patient successfully recovered from the severe condition caused by bone marrow metastases of CAC without any particular critical adverse events of antiandrogen therapy.

Androgens such as testosterone are secreted mainly from the testes, and some are also secreted from the adrenal glands. Androgens function on target organs such as the prostate via the binding to ARs. Antiandrogens suppress the action of androgens in target organs ${ }^{[16]}$. Luteinizing hormone-releasing hormone antagonists inhibit LH secretion from the pituitary gland and subsequently inhibit testosterone secretion from the testes. Androgen antagonists inhibit the activity of androgen from both testes and adrenal glands. Combination androgen blockade (CAB) using a nonsteroid antiandrogen bicaltamide and an luteinizing hormone-releasing hormone antagonist degarelix inhibits the activity of androgen secreted both from the testes and the adrenal glands. The efficacy of $\mathrm{CAB}$ was assessed in the treatment of prostate cancer, and a benefit of $C A B$ compared with castration alone was shown in a meta-analysis ${ }^{[17]}$. Therefore, 
hormonal therapy such as CAB is recognized as the first-choice systemic therapy for metastatic prostate cancer. Because the AR has been reported to be expressed in $60 \%-85 \%$ of breast cancers $^{[18]}$ and salivary duct carcinoma ${ }^{[19-21]}$, there have been several studies of antiandrogen therapy for AR-positive cancers other than prostate cancer. A phase II study of bicalutamide for 26 AR-positive, estrogen receptor-negative metastatic breast cancer patients showed that $19 \%$ of them maintained stable disease for more than 6 months ${ }^{[22]}$. A phase II study of CAB for 36 AR-positive salivary gland carcinoma patients showed that the response rate was $41.7 \%$, and median overall survival was 30.5 months ${ }^{[23]}$. No serious adverse events were reported in both studies. Although no comparisons between antiandrogen therapy and chemotherapy have been reported, the efficacy and safety of CAB for AR-positive tumors have been suggested.

The AR is expressed not only in normal prostate glands and salivary glands, but also in normal apocrine glands. Similar to the functional role of androgens in the prostate gland, androgens have also been suggested to modulate normal apocrine gland function $^{[24]}$. Therefore, AR-expressing CACs might be sensitive to antiandrogen therapy because of the dependency on androgen for their growth or maintenance. The efficacy of hormonal therapy including $\mathrm{CAB}$ varied according to the origin of $\mathrm{AR}$ positive tumors. This is possibly because tumor cell growth and survival might depend on AR-signaling differently. AR-positive cases have been reported to account for $64 \%-100 \%$ of CAC cases $^{[7,25,26]}$. The present patient also had AR-positive CAC, and $\mathrm{CAB}$ showed obvious efficacy without serious adverse events.

\section{Conclusion}

In the present study, antiandrogen therapy for a patient harboring AR-positive CAC with bone and bone marrow metastases resulted in a good clinical course. Although there was no previous case report that showed favorable results of hormonal therapy for $\mathrm{CAC}$, this is the first report demonstrating the efficacy and safety profile of antiandrogen therapy for AR-positive CAC. Further analyses of this therapy are needed to establish a standard treatment strategy for AR-positive CAC.

\section{Ethical approval}

Written informed consent was obtained from the patient for induction of the therapy and publication of this case report and accompanying images. A copy of the written consent is available for review by the Editor-in-Chief of this journal on request.

\section{Source of funding}

None declared.

\section{Author contribution}

T.I. have contributed to treatment of the patient and writing the manuscript. M.I., Y.S., K.T., H.A., H.K., and K.A. contributed to supervise the treatment of the patient. H.Y. and Y.O. contributed to pathological diagnosis. E.B. is a corresponding author and contributed to supervise the treatment and the writing of the final manuscript.

\section{Conflict of interest disclosure}

E.B., the corresponding auther, received speaker's bureau and grant from Takeda Pharma and Daiichi Sankyo. The remaining authors declare that they have no financial conflict of interest with regard to the content of this report.

\section{Research registration unique identifying number (UIN)}

Not applicable.

\section{Guarantor}

The guarantor is that individual who accepts full responsibility for the work and/or the conduct of the study, had access to the data, and controlled the decision to publish.

\section{References}

[1] Hollowell KL, Agle SC, Zervos EE, et al. Cutaneous apocrine adenocarcinoma: defining epidemiology, outcomes, and optimal therapy for a rare neoplasm. J Surg Oncol 2012;105:415-9.

[2] Mezger J, Remberger K, Schalhorn A, et al. Treatment of metastatic sweat gland carcinoma by a four drug combination chemotherapy: response in two cases. Med Oncol Tumor Phamacother 1986;3:29-34.

[3] Bellman B, Gregory NA, Silbers D, et al. Sweat gland carcinoma with metastasis to the skin: response to 5 -fluorouracil chemotherapy. Cutis 1995;55:221-4.

[4] Morabito A, Bevilacqua P, Vitale S, et al. Clinical management of a case of recurrent apocrine gland carcinoma of the scalp: efficacy of a chemotherapy schedule with methotrexate and bleomycin. Tumori 2000;86:472-4.

[5] Hidaka T, Fujimura T, Watabe A, et al. Successful treatment of HER2-positive metastatic apocrine carcinoma of the skin with lapatinib and capecitabine. Acta Derm Venereol 2012;92:654-5.

[6] Rogatsch M, Schmid J, Lax S, et al. Metastatic cutaneous apocrine adenocarcinoma responsive to the programmed cell death protein 1 inhibitor pembrolizumab. Eur J Cancer 2018;90:146-8.

[7] Piris A, Peng Y, Boussahmain C, et al. Cutaneous and mammary apocrine carcinomas have different immunoprofiles. Hum Pathol 2014;45:320-6.

[8] Lehmann BD, Bauer JA, Chen X, et al. Identification of human triplenegative breast cancer subtypes and preclinical models for selection of targeted therapies. J Clin Invest 2011;121:2750-67.

[9] Gallerani E, Ciriolo M, Rossini C, et al. Axillary apocrine carcinoma with brain metastasis. J Clin Oncol 2007;25:5655-6.

[10] Yu S, Jicong G, Yiping L, et al. Axillary apocrine carcinoma with bone metastasis: first report with bone scan image. Ann Clin Case Rep 2017;2:1392.

[11] Wolff AC, Hammond ME, Hicks DG, et al. Recommendation for human epidermal growth factor receptor 2 testing in breast cancer: American Society of Clinical Oncology/College of American Pathologists clinical practice guideline update. J Clin Oncol 2013;31:3997-4013.

[12] Agha RA, Borrelli MR, Farwana R, et al. For the SCARE Group. The SCARE 2018 Statement: Updating Consensus Surgical CAse REport (SCARE) Guidelines. Int J Surg 2018;60:132-6.

[13] Pucevich B, Catinchi-Jaime S, Ho J, et al. Invasive primary ductal apocrine adenocarcinoma of axilla: a case report with immunohistochemical profiling and a review of literature. Dermatol Online J 2008;14:5.

[14] Katagiri Y, Ansai S. Two cases of cutaneous apocrine ductal carcinoma of the axilla. case report and review of the literature. Dermatology 1999; 199:322-7.

[15] Cham PM, Niehans GA, Foman N, et al. Primary cutaneous apocrine carcinoma presenting as carcinoma erysipeloides. Br J Dermatol 2008;158:194-6.

[16] Knudsen BS, Vasioukhin V. Mechanisms of prostate cancer initiation and progression. Adv Cancer Res 2010;109:1-50.

[17] Prostate Cancer Trialists' Collaborative Group. Maximum androgen blockade in advanced prostate cancer: an overview of the randomized trials. Lancet 2000;355:1491-8.

[18] Collins LC, Cole KS, Marotti JD, et al. Androgen receptor expression in breast cancer in relation to molecular phenotype: results from the Nurses' Health Study. Mod Pathol 2011;24:924-31. 
[19] Mitani Y, Rao PH, Maity SN, et al. Alterations associated with androgen receptor gene activation in salivary duct carcinoma of both sexes: potential therapeutic ramifications. Clin Cancer Res 2014;20:6570-81.

[20] Dalin MG, Desrichard A, Katabi N, et al. Comprehensive molecular characterization of salivary duct carcinoma reveals actionable targets and similarity to apocrine breast cancer. Clin Cancer Res 2016;22:4623-33.

[21] Masubuchi T, Tada Y, Maruya S, et al. Clinicopathological significance of androgen receptor, HER2, Ki-67 and EGFR expressions in salivary duct carcinoma. Int J Clin Oncol 2015;20:35-44.

[22] Gucalp A, Tolaney S, Isakoff SJ, et al. Phase II Trials of bicalutamide in patients with androgen receptor-positive, estrogen receptor-negative metastatic breast cancer. Cancer Res 2013;19:5505-12.
[23] Fushimi C, Tada Y, Takahashi H, et al. A prospective phase II study of combined androgen blockade in patients with androgen receptor-positive metastatic or locally advanced unresectable salivary gland carcinoma. Ann Oncol 2018;29:979-84.

[24] Choudhry R, Hodgins MB, Van dear Kwast TH, et al. Localization of androgen receptors in human skin by immunohistochemistry: implications for the hormonal regulation of hair growth, sebaceous glands and sweat glands. J Endocrinol 1992;133:467-75.

[25] Robson A, Lazar AJ, Ben Nagi J, et al. Primary cutaneous apocrine carcinoma: a clinico-pathologic analysis of 24 cases. Am J Surg Pathol 2008;32:682-90.

[26] Long LP, Dias-Santagata D, Pawlak AC, et al. Apocrine-eccrine carcinomas: molecular and immunohistochemical analyses. PLoS One 2012;7:e47290. 